\title{
Supplement to "Lignin oxidation products as a vegetation proxy in stalagmite and drip water samples from the Herbstlabyrinth, Germany"
}

I. Heidke, D. Scholz, T. Hoffmann

S1 Acid-to-aldehyde ratios in the drip water samples

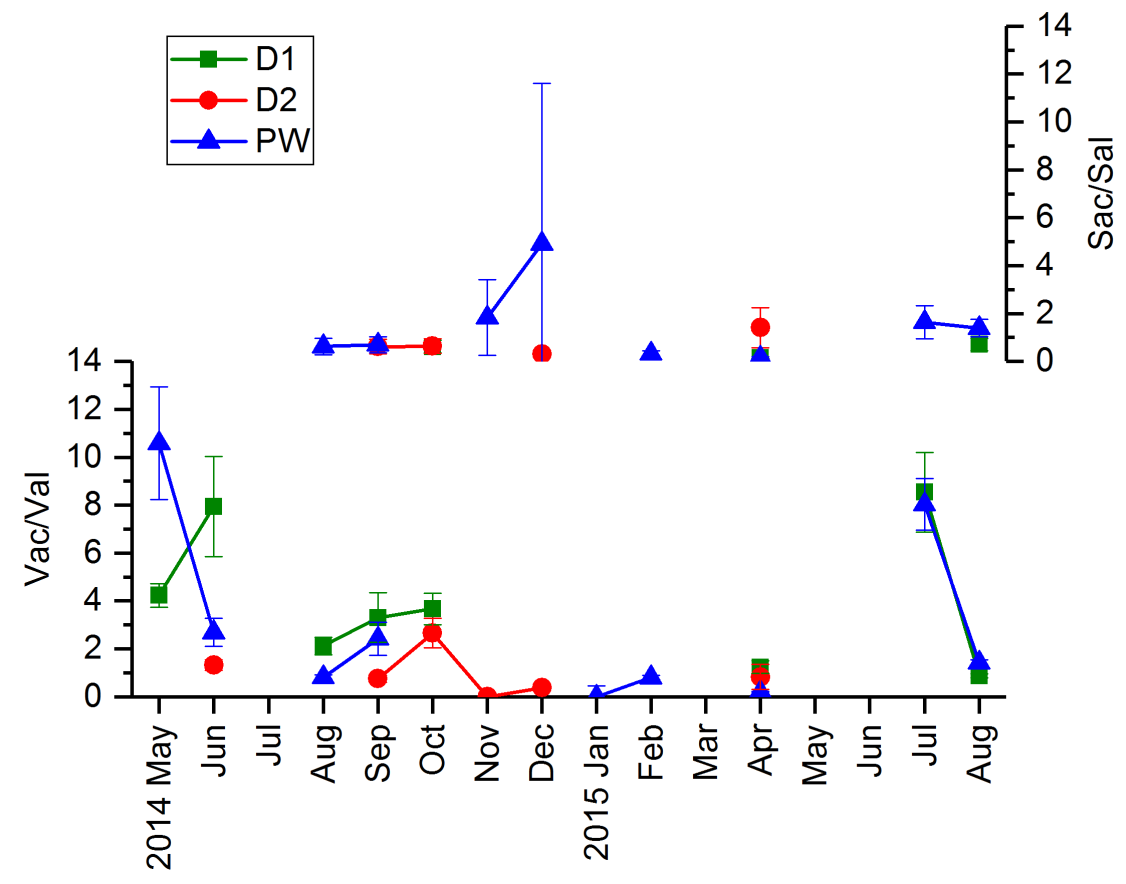

Figure S1. Vac/Val and Sac/Sal ratios in the drip water.

For the fast drip site $D 1$, the values range from $0.9-8.5$ for Vac/Val and 0.2-0.7 for Sac/Sal. For the slow drip site $D 2$, the values are 0.0-2.7 for $\mathrm{Vac} / \mathrm{Val}$ and 0.3-0.6 (1.4 with high uncertainty) for Sac/Sal, and for the pool water $P W, 0.0-10.6$ for $\mathrm{Vac} / \mathrm{Val}$ and 0.2-1.8 (4.9 with high uncertainty) for Sac/Sal. 


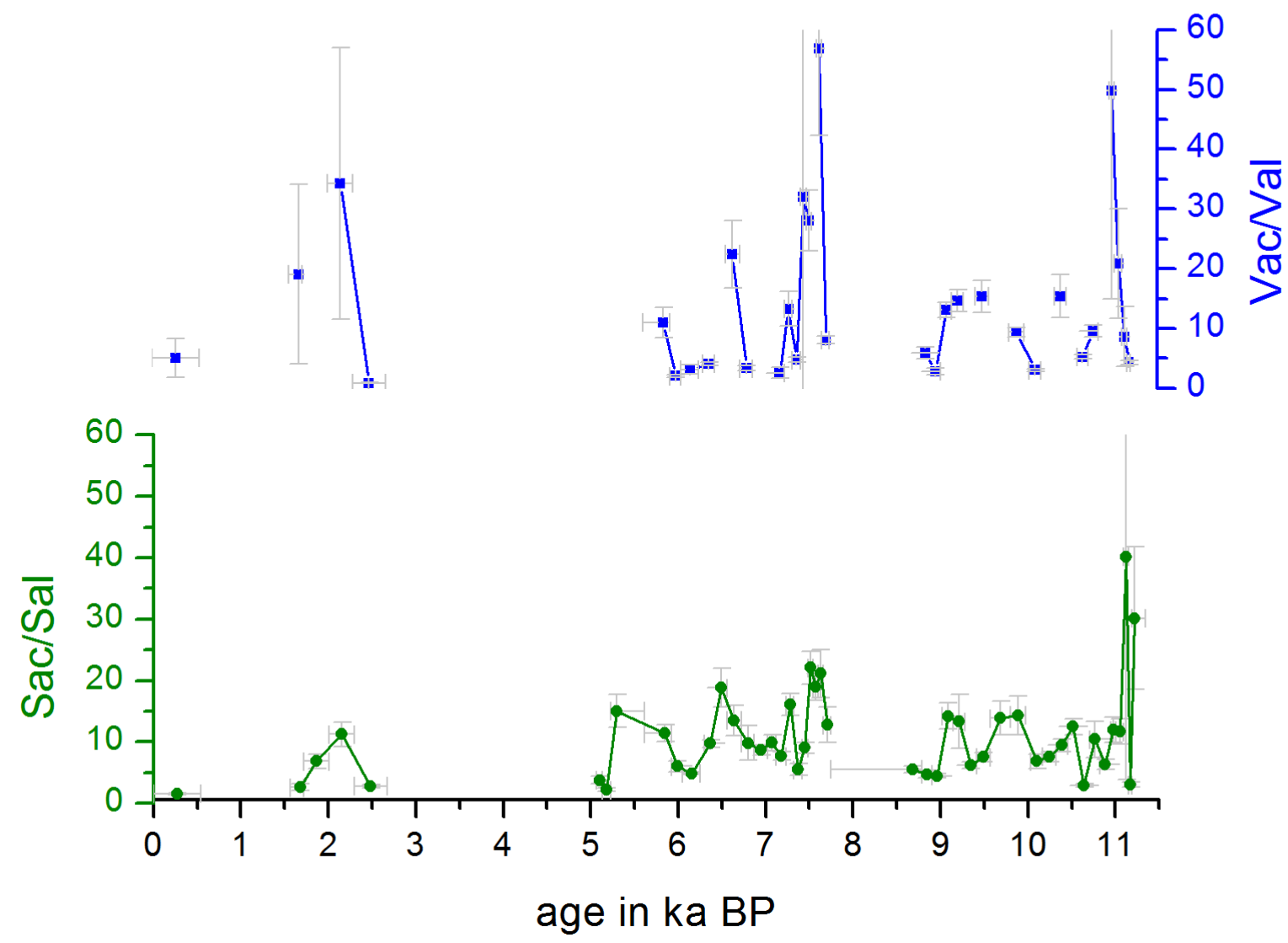

Figure S2. Ratios of syringic acid to syringaldehyde (Sac/Sal) and vanillic acid to vanillin (Vac/Val) in plotted against the age of the stalagmite.

The ratio of Vac/Val in the stalagmite samples ranges from 0.9 to 57, and the ratio of Sac/Sal ranges from 1.6 to 40. 
S3 Correlation coefficients for the older part of the stalagmite

The correlation coefficients for the older part of the stalagmite are shown in table S1 on page S4. 


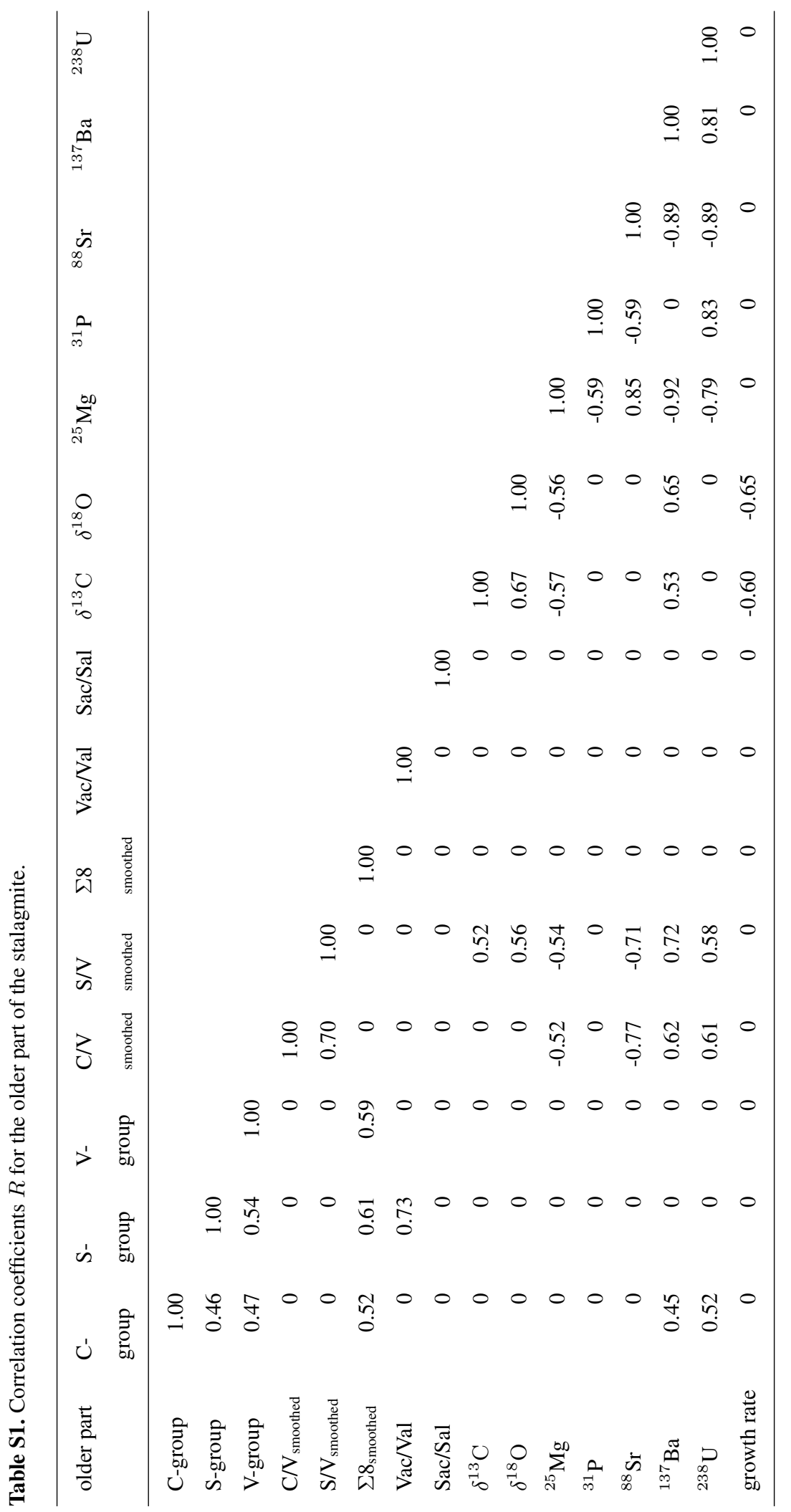


S4 Principal component analysis of LOPs, trace elements and stabel isotopes in the stalagmite samples

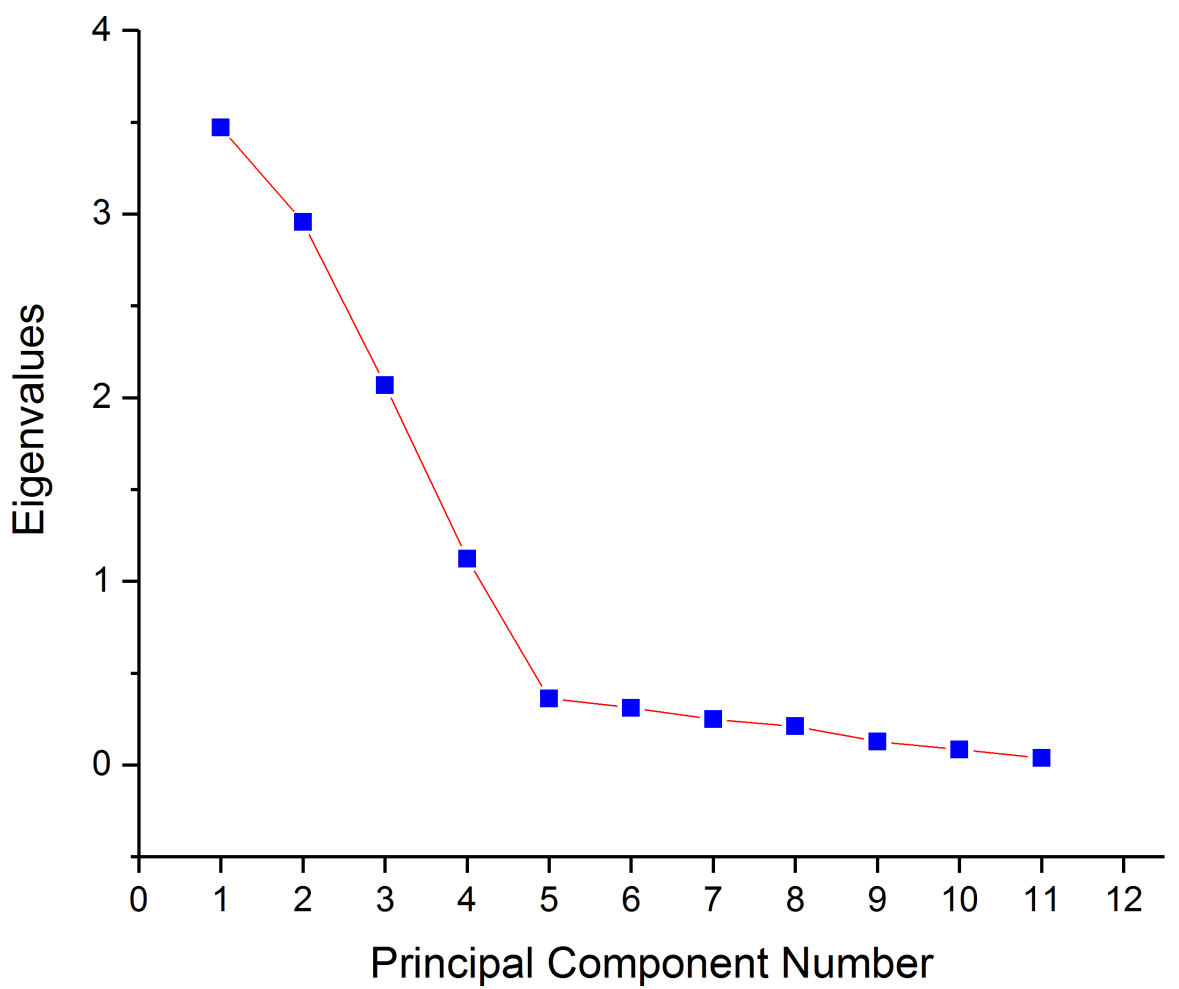

Figure S3. Scree plot showing the eigenvalues of the principal components.

PC3 is composed of $\delta^{18} \mathrm{O}, \delta^{13} \mathrm{C}$ and $\mathrm{Ba}$ with positive loadings and $\mathrm{Mg}$ and the growth rate with negative loadings. PC4 is composed of the growth rate, $\delta^{13} \mathrm{C}$ and $\Sigma 8$ with positive loadings. 


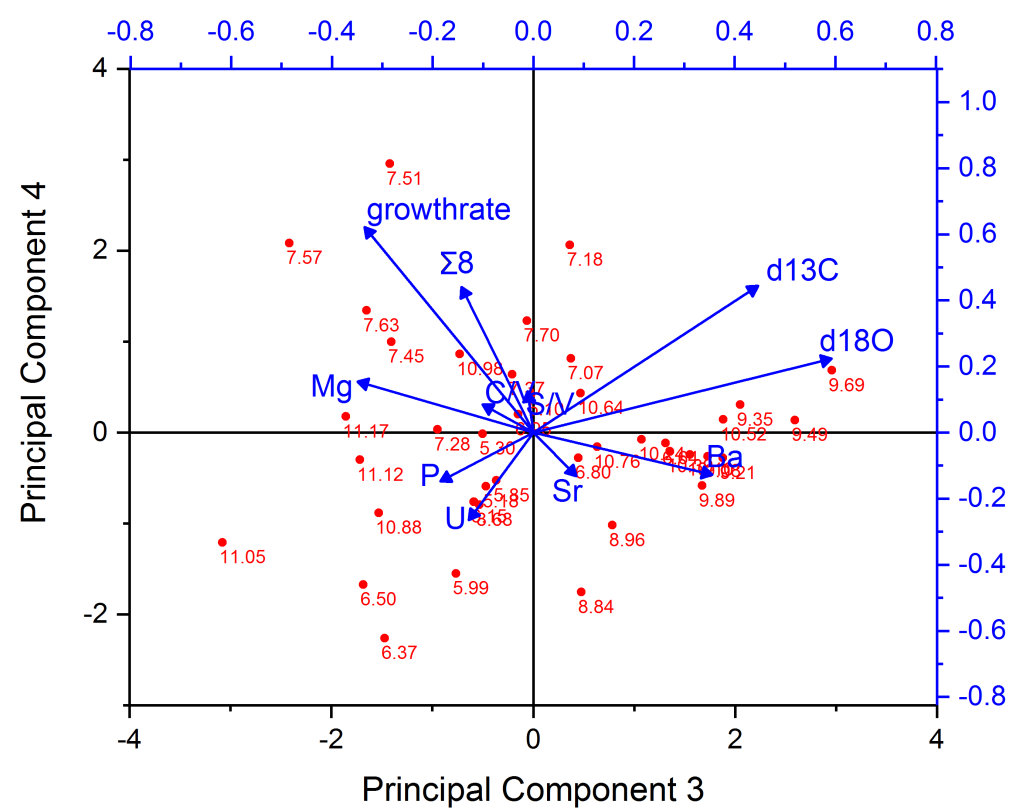

Figure S4. Third (PC3) and fourth (PC4) principal component of the principal component analysis with the variables $\Sigma 8, \mathrm{C} / \mathrm{V}, \mathrm{S} / \mathrm{V}$, trace elements, growth rate and stable isotopes. The red dots are the scores of the individual samples, labeled with their age in ka BP. 